\section{Prenatal diagnosis of hypophosphatasia congenita using ultrasonography}

\author{
Ashwitha Guguloth', Yashant Aswani' ${ }^{2}$ Karan Manoj Anandpara² \\ 'Department of Radiology, Bangalore Medical College and Research Institute, Bangalore; \\ ${ }^{2}$ Department of Radiology, Seth G S Medical College and KEM Hospital, Mumbai, India
}

Congenital hypophosphatasia is a rare fatal skeletal dysplasia. Antenatal determinants of lethality include small thoracic circumference with pulmonary hypoplasia and severe micromelia. These features were present in the fetus of a 25 -year-old female who came for an anomaly scan in her second trimester of pregnancy. Additional findings of generalized demineralization and osteochondral spurs led to the diagnosis of hypophosphatasia congenita. The pregnancy was terminated, and the findings were confirmed on autopsy. Common differential diagnoses with clues to diagnose the above mentioned condition have been discussed here. Early and accurate detection of this medical condition is important as no treatment has been established for this condition. Therefore, antenatal ultrasonography helps in diagnosing and decision making with respect to the current pregnancy and lays the foundation for the genetic counseling of the couple.

Keywords: Hypophosphatasia; Exostoses; Ultrasonography, prenatal

\section{Introduction}

Hypophosphatasia is due to an inborn deficiency of the tissue non-specific alkaline phosphatase (TSALP) gene in osteoblasts and chondrocytes that impairs bone mineralization [1]. It is an inherited skeletal dysplasia with a variable inheritance pattern. There are other forms of hypophosphatasia, but the congenital form carries a mortality rate of $100 \%$ [1]. It shows a severe deficiency of serum and tissue alkaline phosphatase levels and excessive urinary excretion of phosphoethanolamine [1]. In spite of the technical advancements, antenatal ultrasonography is the first tool used for diagnosing and directing the appropriate molecular investigation $[2,3]$. The accuracy of ultrasonography for the diagnosis of lethality is as high as $95 \%$ [4] to $96.8 \%$ [5], which helps decision making with respect to the current pregnancy and delivery. However, here, we would like to emphasize that a specific diagnosis is achieved only in approximately $55 \%$ of the patients [2]. In the present case, the diagnosis of hypophosphatasia congenita $(\mathrm{HC})$ was based on the typical features of this condition and, in rare cases, described pathognomonic osteochondral spurs [6]. Therefore, we highlight the importance of familiarity with antenatal imaging findings of this lethal condition in avoiding maternal morbidity, decreasing the corresponding financial burden on the patient, and providing genetic counseling and help for planning the next pregnancy.

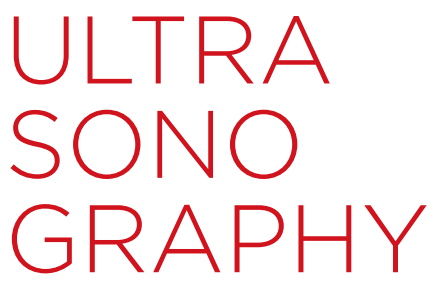

\section{CASE REPORT}

http://dx.doi.org/10.14366/usg.15008 pISSN: 2288-5919 - elSSN: 2288-5943 Ultrasonography 2016;35:83-86

Received: January 28, 2015

Revised: March 17, 2015

Accepted: March 26, 2015

Correspondence to:

Karan Manoj Anandpara, MD, Department of Radiology, Seth G S Medical College and KEM Hospital, Acharya Donde Marg, Parel, Mumbai 400012, India

Tel. +91-98928-53986

Fax. +91-22-24136051

E-mail: karananandpara@gmail.com

This is an Open Access article distributed under the terms of the Creative Commons Attribution NonCommercial License (http://creativecommons.org/ licenses/by-nc/3.0/) which permits unrestricted noncommercial use, distribution, and reproduction in any medium, provided the original work is properly cited.

Copyright (C) 2016 Korean Society of Ultrasound in Medicine (KSUM)

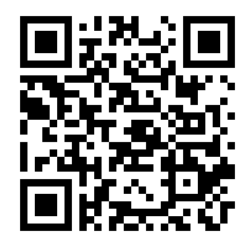

How to cite this article:

Guguloth A, Aswani Y, Anandpara KM. Prenatal diagnosis of hypophosphatasia congenita using ultrasonography. Ultrasonography. 2016 Jan;35(1):83-86. 


\section{Case Report}

A 25-year-old female, gravida 4, para 1, with a history of two previous first trimester abortions and one living male child, came to our services for routine antenatal care; at that time, she had been married to one of her second-degree cousins for 6 years. She was advised to undergo a first trimester dating scan followed by an anomaly scan in the second trimester. The second trimester scan was performed at 19 weeks (according to her last menstrual period) and revealed a single live intrauterine fetus with unstable lie and mild polyhydramnios (amniotic fluid index, $27 \mathrm{~cm}$ ). The fetal parameters of biparietal diameter (47 mm corresponding to 19.9 weeks), head circumference (170 mm corresponding to 19 weeks 4 days), and abdominal circumference (150 mm corresponding to 19 weeks) corresponded to a gestational age of 19 weeks 4 days. Bilateral femora were short $(10.1 \mathrm{~mm}$ and $10.2 \mathrm{~mm}$, corresponding to a gestational age of 12-13 weeks). The humeri on both sides were short as well $(10 \mathrm{~mm}$ and $9.9 \mathrm{~mm}$, respectively, corresponding to a gestational age of 12 weeks). The femora and the humeri measured below the fifth percentile. The tibia, fibula, ulna, and radius could

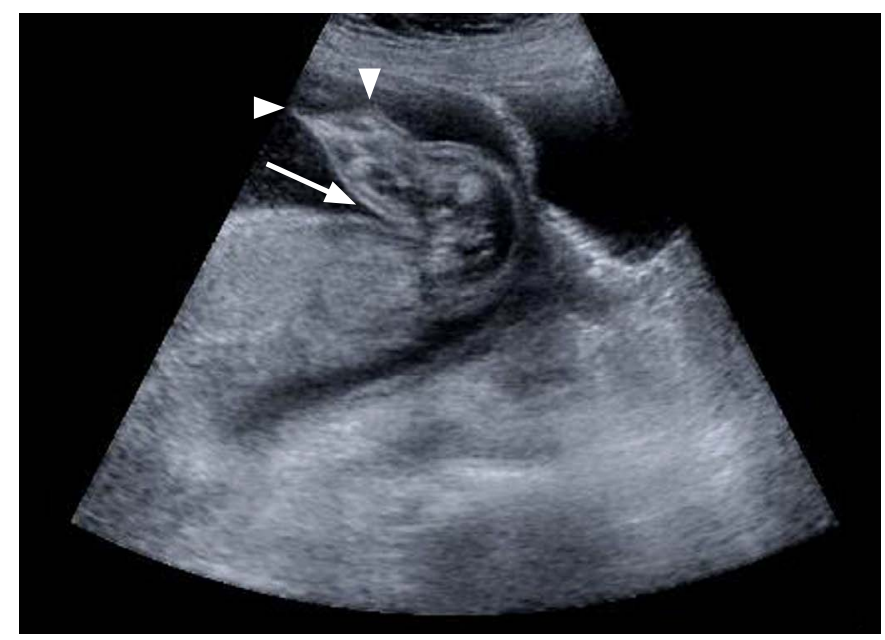

A

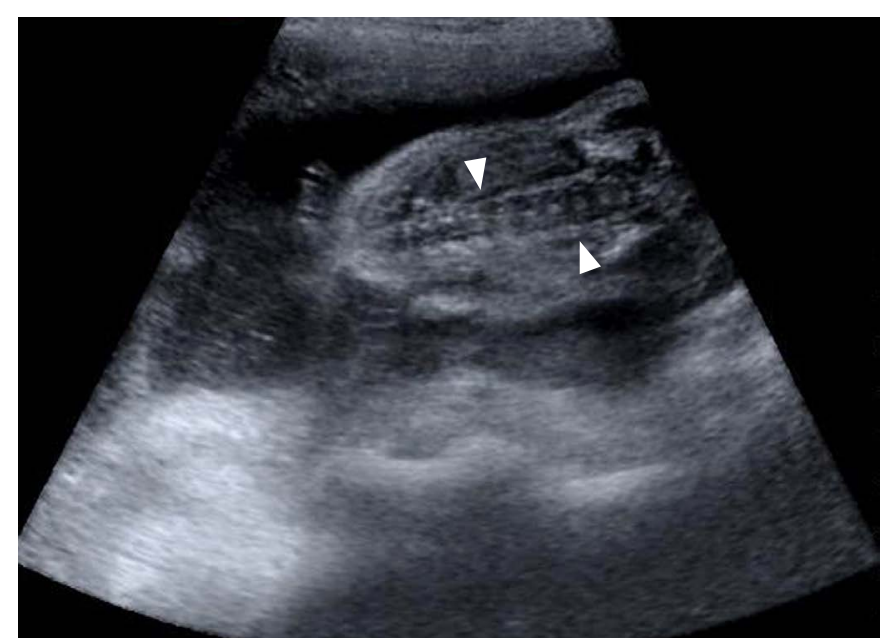

C

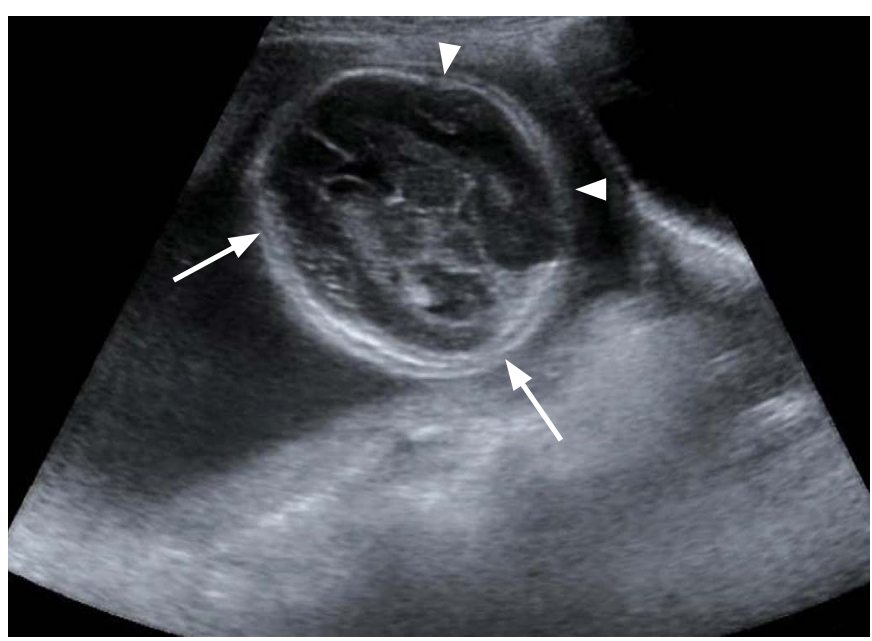

B

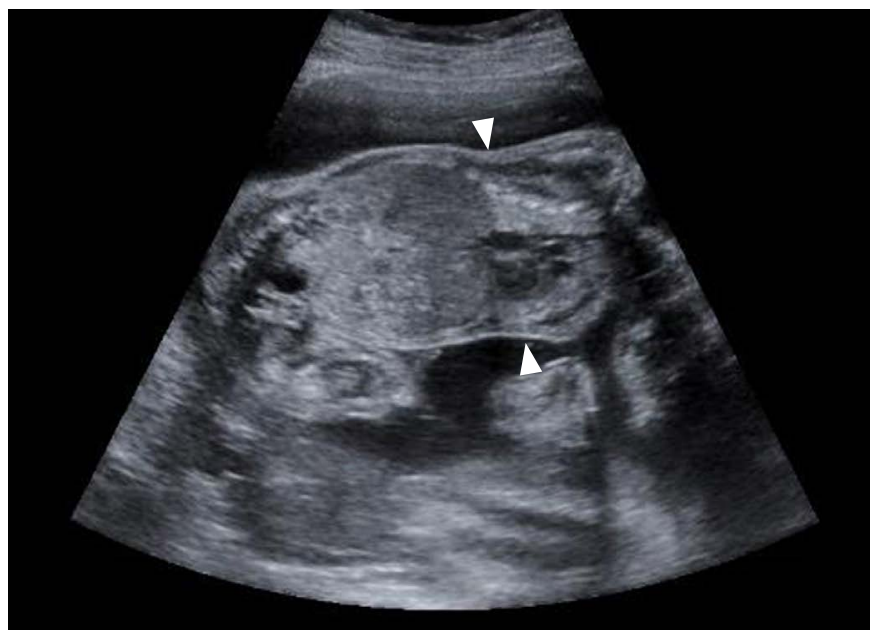

D

Fig. 1. Prenatal ultrasonography of a fetus with hypophosphatasia at the gestational age of 19 weeks.

A. Parasagittal ultrasonography demonstrates the flexion attitude at the hips (arrow). The femora are not well visualized, and demarcation with the tibia-fibula is difficult to identify. Also noted are spurs at the distal aspect of the lower limb (arrowheads). Compare the spurs in the gross pathological specimen shown in Fig. 2A. The limbs are extremely short with contractures at both knees. B. The calvarium looks demineralized with a clear visualization of structures in the near field (arrowheads) as well as the far field (arrows). In an otherwise normally ossified skull, the artifacts caused by shadowing hinder the near-field visualization. C. Vertebral body mineralization also seems to be decreased (arrowheads). D. The sonogram reveals a normal trunk length. The fetal thorax (arrowheads) is narrow with a ratio of the thoracic diameter to the abdominal diameter of 0.7 . 


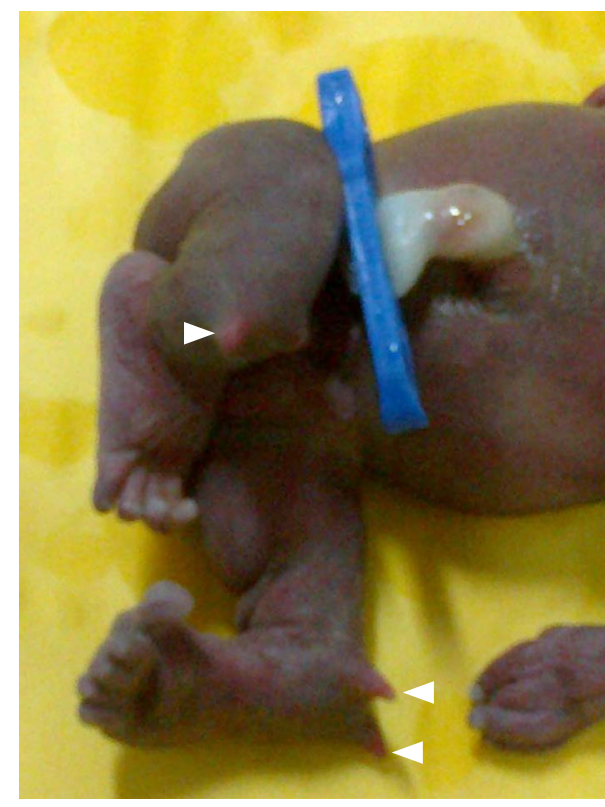

A

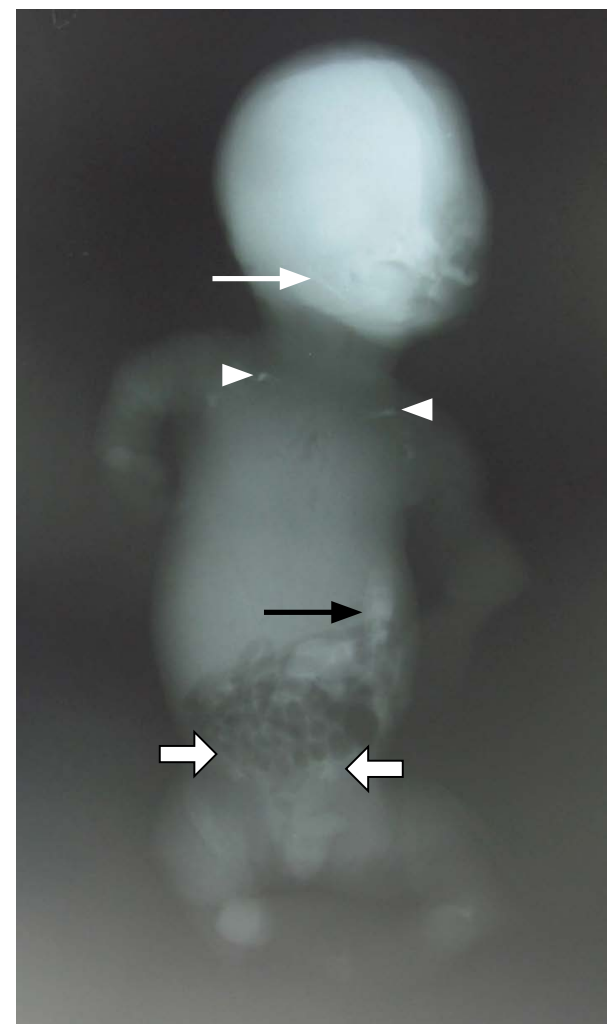

B

Fig. 2. The gross specimen photograph and the infantogram of the fetus with hypophosphatasia congenita delivered at a gestational age of 19 weeks.

A. Multiple skin-covered osteochondral spurs (called Bowdler spurs) (arrowheads) are depicted around the anterior aspect of both knee joints. $B$. The infantogram demonstrates the near-complete absence of skeletal mineralization except for the bilateral clavicle (arrowheads), mandibles (white arrow), and iliac bones (thick white arrows). The radiodensity noted over the lower abdominal region is the clamp for the umbilical stump (black arrow).

not be measured on either side because they were sonolucent. The demarcation between the forearm and arm and the leg and thigh (Fig. 1A) was difficult to identify. There was no evidence of fracture or callus formation. The cranium showed no acoustic shadowing (Fig. 1B) and was deformable upon the application of pressure by using the transducer. The vertebral bodies revealed decreased mineralization (Fig. 1C). The fetal thorax was extremely narrow with a ratio of the thoracic diameter to the abdominal diameter of 0.7 (Fig. 1D). However, the trunk length was normal $(3.5 \mathrm{~cm})$. Furthermore, we observed bone spurs in the lower limbs (Fig. 1A). No abnormal skull shape, neural tube defects, or cardiac defects were noted. The fundic bubble, both kidneys, and urinary bladder were unremarkable. The three-vessel umbilical cord was normal, and the placenta exhibited grade I maturity.

Depending on the abovementioned findings, a diagnosis of $\mathrm{HC}$ was made. The serum alkaline phosphatase levels and DNA analysis for the identification of the TSALP gene were not performed for financial reasons. The woman was counseled about the natural course of the disease and its pattern of inheritance, and consent was taken for the termination of pregnancy. A live male baby, weighing $500 \mathrm{~g}$, was delivered. Immediate neonatal resuscitation was instituted, but the baby succumbed to death in a couple of minutes.

Gross findings (Fig. 2A) included soft and pliable skull, narrow thorax, and micromelia of the bilateral upper and lower limbs. A slight overlap of the digits of both hands was noted. However, the feet appeared normal. Knee and elbow contractures were also observed. Multiple skin-covered osteochondral spurs were observed at the elbow and knee joints. An infantogram was taken; it demonstrated a near-complete absence of the mineralization of the entire skeleton except for the mandible, bilateral clavicles, and iliac bones (Fig. 2B). These features were consistent with the antenatal diagnosis of $\mathrm{HC}$.

\section{Discussion}

Hypophosphatasia, first described by Rathbun in 1948 [1], occurs due to missense mutations in the TSALP gene leading to variable residual enzymatic activity and thus, an extremely high phenotypic heterogeneity. Hypophosphatasia is subdivided into six clinical types according to the age of onset: (1) perinatal/neonatal (lethal), (2) prenatal benign, (3) infantile, (4) childhood, (5) adult, and (6) odontohypophosphatasia [7]. The clinical spectrum varies from a recessively inherited perinatal lethal variant to the late onset dominant form of odontohypophosphatasia, with short stature and premature shedding of teeth. The perinatal form, also known as neonatal hypophosphatasia or HC, is a lethal subtype 
of hypophosphatasia [2]. The pattern of inheritance is autosomal recessive and has a low incidence of approximately 1 in 100,000 births [2].

Typical findings of $\mathrm{HC}$ include severe micromelia, decreased thoracic circumference (the hallmark of fatal osseous dysplasia), and a normal trunk length $[2,3,8]$. Demineralization on account of a variable enzyme deficiency may be patchy or generalized with bowing of bones. However, fractures are occasional. The bones may appear thin and delicate or may appear to be entirely absent at times [2]. The skull size is typically normal [2], and the cranial vault is compressible under gentle transducer pressure. Skin-covered osteochondral spurs (Bowdler spurs) protruding from midshafts or around the elbow or knee joints may be observed. Presence of these spurs is diagnostic for hypophosphatasia [6]. Other findings include apnea, seizures, and marked shortening of the long bones. HC is associated with either stillbirth or death in the early neonatal period due to intracranial hemorrhage or respiratory insufficiency secondary to poorly developed ribs and reduced thoracic cavity volume [2].

HC shows a considerable overlap with osteogenesis imperfecta II and achondrogenesis (the other lethal skeletal dysplasias) $[2,3,8]$, and therefore, a specific antenatal diagnosis can be difficult at times. Presence of thickened, wavy bones due to repetitive callus formation in fragile, fracture-susceptible bones with diffuse demineralization in the former and macrocrania, reduced trunk length, and vertebral demineralization sparing the posterior elements in the latter may aid in arriving at a diagnosis $[2,3,8]$.

There is no treatment for $\mathrm{HC}$, and hence, its prenatal diagnosis is crucial [7]. The prenatal diagnosis may be established by ultrasonography, which can further guide molecular investigations. The ultrasound diagnosis is highly accurate $(95 \%)[2,4]$ for lethal skeletal dysplasias, and given the cost of molecular analysis, the ultrasound diagnosis is the fundamental tool for the diagnosis for these conditions. Prenatal determination of lethality may be specific in only $55 \%$ of the cases $[2,4]$; nonetheless, it is crucial as it provides an opportunity for pregnancy termination and planning of future pregnancies. Biochemical analysis demonstrates a low serum level of alkaline phosphatase and increased urinary concentration of phosphoethanolamine in both the plasma and the urine [9]. However, Moore et al. [9] have described cases of hypophosphatasia with normal enzymatic activities. Molecular analysis in the form of DNA analysis is also available. Since $\mathrm{HC}$ has an autosomal recessive pattern of inheritance with a $25 \%$ chance of recurrence in the next pregnancy, genetic counseling is important for all parents with a previously affected child. However, the genetic counseling of $\mathrm{HC}$ is complicated by the variability of inheritance patterns (autosomal dominant or autosomal recessive) and by an incomplete penetrance of the trait. Therefore, we believe that it is worthwhile to discuss prenatal testing for the perinatal lethal form of $\mathrm{HC}$ for future pregnancies in these parents [7]. There is no curative treatment for hypophosphatasia, and the availability of enzyme replacement therapy will certainly be a promising therapeutic tool [7].

$\mathrm{HC}$ is a rare fatal osseous dysplasia for which treatment is not available. Hence, the detection of index cases and prenatal diagnosis in subsequent pregnancies is very important. This additionally avoids maternal morbidity and increased financial burden on the family.

ORCID: Ashwitha Guguloth: http://orcid.org/0000-0002-0351-4352; Yashant Aswani: http://orcid.org/0000-0001-6994-8826; Karan Manoj Anandpara: http://orcid.org/00000001-9451-7577

\section{Conflict of Interest}

No potential conflict of interest relevant to this article was reported.

\section{References}

1. Mumm S, Jones J, Finnegan P, Whyte MP. Hypophosphatasia: molecular diagnosis of Rathbun's original case. J Bone Miner Res 2001;16:1724-1727.

2. Glanc $P$, Chitayat $D$, Unger $S$. The fetal musculoskeletal system. In: Rumack CM, Wilson SR, Charboneau JW, Levine D, eds. Diagnostic ultrasound. 4th ed. Philadelphia: Elsevier Mosby, 2011;1389-1423.

3. Schramm T, Gloning KP, Minderer S, Daumer-Haas C, Hortnagel $\mathrm{K}$, Nerlich $\mathrm{A}$, et al. Prenatal sonographic diagnosis of skeletal dysplasias. Ultrasound Obstet Gynecol 2009;34:160-170.

4. Tretter AE, Saunders RC, Meyers CM, Dungan JS, Grumbach K, Sun CC, et al. Antenatal diagnosis of lethal skeletal dysplasias. Am J Med Genet 1998;75:518-522.

5. Krakow D, Alanay Y, Rimoin LP, Lin V, Wilcox WR, Lachman RS, et al. Evaluation of prenatal-onset osteochondrodysplasias by ultrasonography: a retrospective and prospective analysis. Am J Med Genet A 2008;146A:1917-1924.

6. Vandevijver $N$, De Die-Smulders $C E$, Offermans JP, Van Der Linden ES, Arends JW, Sastrowijoto SH, et al. Lethal hypophosphatasia, spur type: case report and fetopathological study. Genet Couns 1998;9:205-209.

7. Mornet E. Hypophosphatasia. Orphanet J Rare Dis 2007;2:40.

8. Zankl A, Mornet E, Wong S. Specific ultrasonographic features of perinatal lethal hypophosphatasia. Am J Med Genet A 2008;146A:1200-1204.

9. Moore CA, Wappner RS, Coburn SP, Mulivor RA, Fedde KN, Whyte MP. Pseudohypophosphatasia: clinical, radiographic and biochemical characteristics in a second case. Am J Hum Genet 1990;47(Suppl):A68. 